\title{
Nested Lattice Codes for Arbitrary Continuous Sources and Channels
}

\author{
Aria G. Sahebi and S. Sandeep Pradhan \\ Department of Electrical Engineering and Computer Science, \\ University of Michigan, Ann Arbor, MI 48109, USA. \\ Email: ariaghs@umich.edu, pradhanv@umich.edu
}

\begin{abstract}
In this paper, we show that nested lattice codes achieve the capacity of arbitrary continuous channels with or without non-causal state information at the transmitter. We also show that nested lattice codes are optimal for source coding with or without non-causal side information at the receiver for arbitrary continuous sources. We show the optimality of lattice codes for the Gelfand-Pinsker and Wyner-Ziv problems in their most general settings.
\end{abstract}

\section{INTRODUCTION}

Lattice codes for continuous sources and channels are the analogue of linear codes for discrete sources and channels and play an important role in information theory and communications. Linear/lattice and nested linear/lattice codes have been used in many communication settings to improve upon the existing random coding bounds [2], [10]-[13], [16], [18], [20].

In [2] and [12] the existence of lattice codes satisfying Shannon's bound has been shown. These results have been generalized and the close relation between linear and lattice codes has been pointed out in [13]. In [24], several results regarding lattice quantization noise in high resolution has been derived and the problem of constructing lattices with an arbitrary quantization noise distribution has been studied in [7].

Nested lattice codes were introduced in [26] where the concept of structured binning is presented. Nested linear/lattice codes are important because in many communication problems, specially multi-terminal settings, such codes can be superior in average performance compared to random codes [11]. It has been shown in [25] that nested lattice codes are optimal for the Wyner-Ziv problem when the source and side information are jointly Gaussian. The dual problem of channel coding with state information has been addressed in [4]-[6], [21] and the optimality of lattice codes for Gaussian channels has been shown. In [3], it was shown that random linear codes provide good binning schemes for general Slepian-Wolf coding.

In a recent work [17], it has been shown that nested linear codes are optimal for arbitrary discrete memoryless channels with state information at the transmitter. In this paper we focus on two problems: 1) The point to point channel coding with state information at the encoder (the Gelfand-Pinsker problem [8]) and 2) Lossy source coding with side information at the

This work was supported by NSF grants CCF-0915619 and CCF-1116021. decoder (the Winer-Ziv problem [22], [23]). We consider these two problems in their most general settings i.e. when the source and the channel are arbitrary but memoryless. We use nested lattice codes with joint typicality decoding/encoding rather than lattice decoding. We consider a lattice code ensemble with simpler random dithers and without modulo- $\Lambda$ transformations as used in [25]. We show that in both settings, from an information-theoretic point of view, nested lattice codes are optimal.

The paper is organized as follows: in Section II we present the required preliminaries and introduce our notation. In Section III we show the optimality of nested lattice codes for channels with state information (the Gelfand-Pinsker problem). We briefly present the optimality of nested lattice codes for source coding with side information (the Wyner-Ziv problem) in Section IV and we conclude in Section V.

\section{PREliminaries}

1) Channel Model: We associate two sets $\mathcal{X}$ and $\mathcal{Y}$ with the channel as the channel input and output alphabets. The set of channel states is denoted by $\mathcal{S}$ and it is assumed that the channel state is distributed over $\mathcal{S}$ according to $P_{S}$. When the state of the channel $S$ is $s \in \mathcal{S}$, the input-output relation of the channel is characterized by a transition kernel $W_{Y \mid X S}(y \mid x, s)$ for $x \in \mathcal{X}$ and $y \in \mathcal{Y}$. We assume the state of the channel is known at the transmitter non-causally. The channel is specified by $\left(\mathcal{X}, \mathcal{Y}, \mathcal{S}, P_{S}, W_{Y \mid X S}, w\right)$ where $w$ is the cost function.

2) Source Model: The source is modeled as a discretetime random process $X$ with each sample taking values in a fixed set $\mathcal{X}$ called alphabet. Assume $X$ is distributed jointly with the random variable $S$ according to the measure $P_{X S}$ over $\mathcal{X} \times \mathcal{S}$ where $\mathcal{S}$ is an arbitrary set. We assume that the side information $S$ is known to the receiver non-causally. The reconstruction alphabet is denoted by $\mathcal{U}$ and the quality of reconstruction is measured by a single-letter distortion functions $d: \mathcal{X} \times \mathcal{U} \rightarrow \mathbb{R}^{+}$. We denote such sources by $\left(\mathcal{X}, \mathcal{S}, \mathcal{U}, P_{X S}, d\right)$.

3) Linear and Coset Codes Over $\mathbb{Z}_{p}$ : For a prime number $p$, a linear code over $\mathbb{Z}_{p}$ of length $n$ and rate $R=\frac{k}{n} \log p$ is a collection of $p^{k}$ codewords of length $n$ which is closed under mod- $p$ addition. Any such code can be characterized by its generator matrix $G \in \mathbb{Z}_{p}^{k \times n}$. The set of all message tuples for this code is $\mathbb{Z}_{p}^{k}$ and the set of all codewords is the range of the matrix $G$. The linear encoder maps a message 
tuple $u \in \mathbb{Z}_{p}^{k}$ to the codeword $x$ where $x=u G$ and the operations are done mod- $p$. A coset code over $\mathbb{Z}_{p}$ is a shift of a linear code by a fixed vector. A coset code of length $n$ and rate $R=\frac{k}{n} \log p$ is characterized by its generator matrix $G \in \mathbb{Z}_{p}^{k \times n}$ and it's shift vector (dither) $B \in \mathbb{Z}_{p}^{n}$. The encoding rule for the corresponding coset code is given by $x=u G+B$, where $u$ is the message tuple and $x$ is the codeword.

4) Lattice Codes and Shifted Lattice Codes: A lattice code of length $n$ is a collection of codewords in $\mathbb{R}^{n}$ which is closed under real addition. A shifted lattice code is any translation of a lattice code by a real vector. In this paper, we use coset codes to construct (shifted) lattice codes as follows: Given a coset code $\mathbb{C}$ of length $n$ over $\mathbb{Z}_{p}$ and a step size $\gamma$, define $\Lambda(\mathbb{C}, \gamma, p)=\gamma\left(\mathbb{C}-\frac{p-1}{2}\right)$. Then the corresponding mod- $p$ lattice code $\bar{\Lambda}(\mathbb{C}, \gamma, p)$ is the disjoint union of shifts of $\Lambda$ by vectors in $\gamma p \mathbb{Z}^{n}$. i.e. $\bar{\Lambda}(\mathbb{C}, \gamma, p)=\bigcup_{v \in p \mathbb{Z}^{n}}(\gamma v+\Lambda)$. Note that $\Lambda(\mathbb{C}, \gamma, p) \subseteq \bar{\Lambda}(\mathbb{C}, \gamma, p)$ is a scaled and shifted copy of the linear code $\mathbb{C}$.

5) Nested Linear Codes: A nested linear code consists of two linear codes, with the property than one of the codes (the inner linear code) is a subset of the other code (the outer linear code). For positive integers $k$ and $l$, let the outer and inner codes $\mathbb{C}_{i}$ and $\mathbb{C}_{o}$ be linear codes over $\mathbb{Z}_{p}$ characterized by their generator matrices $G \in \mathbb{Z}_{p}^{l \times n}$ and $G^{\prime} \in \mathbb{Z}_{p}^{(k+l) \times n}$ and their shift vectors $B \in \mathbb{Z}_{p}^{n}$ and $B^{\prime} \in \mathbb{Z}_{p}^{n}$ respectively. Furthermore, assume $G^{\prime}=\left[\begin{array}{c}G \\ \Delta G\end{array}\right]$ for some $\Delta G \in \mathbb{Z}_{p}^{k \times n}$ and $B^{\prime}=B$. In this case,

$$
\begin{aligned}
& \mathbb{C}_{o}=\left\{a G+m \Delta G+B \mid a \in \mathbb{Z}_{p}^{l}, m \in \mathbb{Z}_{p}^{k}\right\}, \\
& \mathbb{C}_{i}=\left\{a G+B \mid a \in \mathbb{Z}_{p}^{l}\right\}
\end{aligned}
$$

It is clear that the inner code is contained in the outer code. Furthermore, the inner code induces a partition of the outer code through its shifts. For $m \in \mathbb{Z}_{p}^{k}$ define the $m$ th bin of $\mathbb{C}_{i}$ in $\mathbb{C}_{o}$ as $\mathbb{B}_{m}=\left\{a G+m \Delta G+B \mid a \in \mathbb{Z}_{p}^{l}\right\}$. The outer code is the disjoint union of all the bins and each bin index $m \in \mathbb{Z}_{p}^{k}$ is considered as a message. We denote a nested linear code by a pair $\left(\mathbb{C}_{i}, \mathbb{C}_{o}\right)$.

6) Nested Lattice Codes: Given a nested linear code $\left(\mathbb{C}_{i}, \mathbb{C}_{o}\right)$ over $\mathbb{Z}_{p}$ and a step size $\gamma$, define

$$
\begin{aligned}
& \Lambda_{i}\left(\mathbb{C}_{i}, \gamma, p\right)=\gamma\left(\mathbb{C}_{i}-\frac{p-1}{2}\right), \\
& \Lambda_{o}\left(\mathbb{C}_{o}, \gamma, p\right)=\gamma\left(\mathbb{C}_{o}-\frac{p-1}{2}\right)
\end{aligned}
$$

Then the corresponding nested lattice code consists of an inner lattice code and an outer lattice code

$$
\begin{aligned}
\bar{\Lambda}_{i}\left(\mathbb{C}_{i}, \gamma, p\right) & =\cup_{v \in p \mathbb{Z}^{n}}\left(\gamma v+\Lambda_{i}\right) \\
\bar{\Lambda}_{o}\left(\mathbb{C}_{o}, \gamma, p\right) & =\cup_{v \in p \mathbb{Z}^{n}}\left(\gamma v+\Lambda_{o}\right)
\end{aligned}
$$

In this case as well, the inner lattice code induces a partition of the outer lattice code. For $m \in \mathbb{Z}_{p}^{k}$, define $\mathfrak{B}_{m}=\gamma\left(\mathbb{B}_{m}-\frac{p-1}{2}\right)$ where $\mathbb{B}_{m}$ is the $m$ th bin of $\mathbb{C}_{i}$ in $\mathbb{C}_{o}$. The $m$ th bin of the inner lattice code in the outer lattice code is defined by:

$$
\overline{\mathfrak{B}}_{m}=\cup_{v \in p \mathbb{Z}^{n}}\left(\gamma v+\mathfrak{B}_{m}\right)
$$

The set of messages consists of the set of all bins of $\bar{\Lambda}_{i}$ in $\bar{\Lambda}_{o}$. We denote a nested lattice code by a pair $\left(\bar{\Lambda}_{i}, \bar{\Lambda}_{o}\right)$.

7) Achievability for Channel Coding: A transmission system with parameters $(n, M, \Gamma, \tau)$ for reliable communication over a given channel $\left(\mathcal{X}, \mathcal{Y}, \mathcal{S}, P_{S}, W_{Y \mid X S}\right)$ with cost function $w: \mathcal{X} \rightarrow \mathbb{R}^{+}$consists of an encoding mapping and a decoding mapping $e: \mathcal{S}^{n} \times\{1,2, \ldots, M\} \rightarrow \mathcal{X}^{n}, f$ : $\mathcal{Y}^{n} \rightarrow\{1,2, \ldots, M\}$ such that for all $m=1,2, \ldots, M$, if $x=\left(x_{1}, \cdots, x_{n}\right)=e(m)$ then $\frac{1}{n} \sum_{i=1}^{n} w\left(x_{i}\right)<\Gamma$ and $\sum_{m=1}^{M} \frac{1}{M} \operatorname{Pr}\left(f\left(Y^{n}\right) \neq m \mid X^{n}=e(m)\right) \leq \tau$.

Given a channel $\left(\mathcal{X}, \mathcal{Y}, \mathcal{S}, f_{S}, f_{Y \mid X S}\right)$, a pair of non negative numbers $(R, W)$ is said to be achievable if for all $\epsilon>0$ and for all sufficiently large $n$, there exists a transmission system for reliable communication with parameters $(n, M, \Gamma, \tau)$ such that $\frac{1}{n} \log M \geq R-\epsilon, \Gamma \leq W+\epsilon$ and $\tau \leq \epsilon$.

8) Achievability for Source Coding: A transmission system with parameters $(n, \Theta, \Delta, \tau)$ for compressing a given source $\left(\mathcal{X}, \mathcal{S}, \mathcal{U}, P_{X S}, d(\cdot)\right)$ consists of an encoding mapping $e: \mathcal{X}^{n} \rightarrow\{1,2, \cdots, \Theta\}$ and a decoding mapping $g: \mathcal{S}^{n} \times$ $\{1,2, \cdots, \Theta\} \rightarrow \mathcal{U}^{n}$ such that $P\left(d\left(X^{n}, g\left(e\left(X^{n}\right)\right)\right)>\Delta\right) \leq$ $\tau$ where $X^{n}$ is the random vector of length $n$ generated by the source. In this transmission system, $n$ denotes the block length, $\log \Theta$ denotes the number of channel uses, $\Delta$ denotes the distortion level and $\tau$ denotes the probability of exceeding the distortion level $\Delta$.

Given a source, a pair of non-negative real numbers $(R, D)$ is said to be achievable if there exists for every $\epsilon>0$, and for all sufficiently large numbers $n$ a transmission system with parameters $(n, \Theta, \Delta, \tau)$ for compressing the source such that $\frac{1}{n} \log \Theta \leq R+\epsilon, \Delta \leq D+\epsilon$ and $\tau \leq \epsilon$.

9) Mutual Information and Kullback-Leibler divergence: Let $Q=\left\{A_{1}, A_{2}, \cdots, A_{r}\right\}$ be a finite measurable partition of $\mathbb{R}^{d}$. For random variables $U$ and $Y$ on $\mathbb{R}^{d}$ with measure $P_{U Y}$ define the quantized random variables $U_{Q}$ and $Y_{Q}$ on $Q$ with measure $P_{U_{Q} Y_{Q}}\left(A_{i}, A_{j}\right)=P_{U Y}\left(A_{i}, A_{j}\right)$. The Kullback-Leibler divergence between $U$ and $Y$ is defined as $D(U \| Y)=\sup _{Q} D\left(U_{Q} \| Y_{Q}\right)$ where $D\left(U_{Q} \| Y_{Q}\right)$ is the discrete Kullback-Leibler divergence and the supremum is taken over all finite partitions $Q$ of $\mathbb{R}^{d}$. Similarly, the mutual information between $U$ and $Y$ is defined as $I(U ; Y)=\sup _{Q} I\left(U_{Q} ; Y_{Q}\right)$ where $I\left(U_{Q} ; Y_{Q}\right)$ is the discrete mutual information between the two random variables and the supremum is taken over all finite partitions $Q$ of $\mathbb{R}^{d}$.

10) Typicality: We use the notion of weak* typicality with Prokhorov metric introduced in [14]. Let $M\left(\mathbb{R}^{d}\right)$ be the set of probability measures on $\mathbb{R}^{d}$. For a subset $A$ of $\mathbb{R}^{d}$ define its $\epsilon$-neighborhood by $A^{\epsilon}=\left\{x \in \mathbb{R}^{d} \mid \exists y \in A\right.$ such that $\| x-$ $y \|<\epsilon\}$ where $\|\cdot\|$ denotes the Euclidean norm in $\mathbb{R}^{d}$. The Prokhorov distance between two probability measures $P_{1}, P_{2} \in M\left(\mathbb{R}^{d}\right)$ is defined as follows:

$$
\begin{aligned}
\pi_{d}\left(P_{1}, P_{2}\right)= & \inf \left\{\epsilon>0 \mid P_{1}(A)>P_{2}\left(A^{\epsilon}\right)+\epsilon\right. \text { and } \\
& \left.P_{2}(A)>P_{1}\left(A^{\epsilon}\right)+\epsilon \quad \forall \text { Borel set } A \text { in } \mathbb{R}^{d}\right\}
\end{aligned}
$$


Consider two random variables $X$ and $Y$ with joint distribution $P_{X Y}(\cdot, \cdot)$ over $\mathcal{X} \times \mathcal{Y} \subseteq \mathbb{R}^{2}$. Let $n$ be an integer and $\epsilon$ be a positive real number. For the sequence pair $(x, y)$ belonging to $\mathcal{X}^{n} \times \mathcal{Y}^{n}$ where $x=\left(x_{1}, \cdots, x_{n}\right)$ and $y=\left(y_{1}, \cdots, y_{n}\right)$ define the empirical joint distribution by

$$
P_{x y}(A, B)=\frac{1}{n} \sum_{i=1}^{n} \mathbb{1}_{\left\{x_{i} \in A, y_{i} \in B\right\}}
$$

for Borel sets $A$ and $B$. Let $P_{x}$ and $P_{y}$ be the corresponding marginal probability measures. It is said that the sequence $x$ is weakly* $\epsilon$-typical with respect to $P_{X}$ if $\pi_{1}\left(P_{X}, P_{x}\right)<\epsilon$. We denote the set of all weakly* $\epsilon$-typical sequences of length $n$ by $A_{\epsilon}^{n}(X)$. Similarly, $x$ and $y$ are said to be jointly weakly* $\epsilon$-typical with respect to $P_{X Y}$ if $\pi_{2}\left(P_{x y}, P_{X Y}\right)<\epsilon$. We denote the set of all weakly* $\epsilon$ typical sequence pairs of length $n$ by $A_{\epsilon}^{n}(X Y)$. We define $A_{\epsilon}^{n}(Y \mid x)=\left\{y \in \mathcal{Y}^{n} \mid(x, y) \in A_{\epsilon}^{n}(X, Y)\right\}$.

11) Notation: In our notation, $O(\epsilon)$ is any function of $\epsilon$ such that $\lim _{\epsilon \rightarrow 0} O(\epsilon)=0$ and for a set $G,|G|$ denotes the cardinality (size) of $G$.

\section{Channel CODING}

We show the achievability of the rate $R=I(U ; Y)-$ $I(U ; S)$ for the Gelfand-Pinsker channel using nested lattice code for $U$.

Theorem III.1. For the channel $\left(\mathcal{X}, \mathcal{Y}, \mathcal{S}, P_{S}, W_{Y \mid X S}\right)$ where $\mathcal{X}, \mathcal{X}, \mathcal{X} \subseteq \mathbb{R}$, let $w: \mathcal{X} \rightarrow \mathbb{R}^{+}$be a continuous cost function. Let $\mathcal{U}$ be an arbitrary set and let $S U X Y$ be distributed over $\mathcal{S} \times \mathcal{U} \times \mathcal{X} \times \mathcal{Y}$ according to $P_{S} P_{U \mid S} W_{X \mid U S} W_{Y \mid S X}$ where $P_{U \mid S}$ and $W_{X \mid U S}$ are such that $\mathbb{E}\{w(X)\} \leq W$. Then the pair $(R, W)$ is achievable using nested lattice codes where $R=I(U ; Y)-I(U ; S)$.

\section{A. Discrete $U$ and Bounded Continuous Cost Function}

In this section we prove the theorem for the case when $U=\hat{U}$ takes values from the discrete set $\gamma\left(\mathbb{Z}_{p}-\frac{p-1}{2}\right)$ where $p$ is a prime and $\gamma$ is a positive number. We use a random coding argument over the ensemble of mod- $p$ lattice codes to prove the achievability. Let $\mathbb{C}_{o}$ and $\mathbb{C}_{i}$ be defined as (1) and (2) where $G$ is a random matrix in $\mathbb{Z}_{p}^{l \times n}, \Delta G$ is a random matrix in $\mathbb{Z}_{p}^{k \times n}$ and $B$ is a random vector in $\mathbb{Z}_{p}^{n}$, all uniformly distributed over their respective domains. Define $\bar{\Lambda}_{i}\left(\mathbb{C}_{i}, \gamma, p\right)$ and $\bar{\Lambda}_{o}\left(\mathbb{C}_{o}, \gamma, p\right)$ accordingly. The ensemble of nested lattice codes consists of all lattices of the form (3) and (4). The set of messages consists of all bins $\mathfrak{B}_{m}$ indexed by $m \in \mathbb{Z}_{p}^{k}$. The encoder observes the message $m \in \mathbb{Z}_{p}^{k}$ and the channel state $s \in \mathcal{S}^{n}$ and looks for a vector $u$ in the $m$ th bin $\mathfrak{B}_{m}$ which is jointly weak* typical with $s$ and encodes the message $m$ to $x$ according to $W_{X \mid S U}$. The encoder declares error if it does not find such a vector.

After receiving $y \in \mathcal{Y}^{n}$, the decoder decodes it to $m \in \mathbb{Z}_{p}^{k}$ if $m$ is the unique tuple such that the $m$ th bin $\mathfrak{B}_{m}$ contains a sequence jointly typical with $y$. Otherwise it declares error.
1) Encoding Error: Let $S^{\prime}=\left[\frac{-\gamma p}{2}, \frac{\gamma p}{2}\right]^{n} \cap \gamma \mathbb{Z}^{n}$. For $a \in$ $\mathbb{Z}_{p}^{k}, m \in \mathbb{Z}_{p}^{l}$, define

$$
g(a, m)=\gamma\left((a G+m \Delta G+B)-\frac{(p-1)}{2}\right)
$$

$g(a, m)$ has the following properties:

Lemma III.2. For $a \in \mathbb{Z}_{p}^{l}$ and $m \in \mathbb{Z}_{p}^{k}, g(a, m)$ is uniformly distributed over $S^{\prime}$.

Proof: Follows from the fact that $B$ is independent of $G$ and $\Delta G$ and is uniformly distributed.

Lemma III.3. For $a, \tilde{a} \in \mathbb{Z}_{p}^{l}$ and $m \in \mathbb{Z}_{p}^{k}$ if $a \neq \tilde{a}$ then $g(a, m)$ and $g(\tilde{a}, m)$ are pairwise independent.

Proof: Follows from a counting argument.

For message $m \in \mathbb{Z}_{p}^{k}$ and state $s \in \mathcal{S}^{n}$, the encoder declares error if there is no sequence in $\mathfrak{B}_{m}$ jointly typical with $s$. Define

$$
\theta(s)=\sum_{u \in \mathfrak{B}_{m}} \mathbb{1}_{\left\{u \in A_{\epsilon}^{n}(\hat{U} \mid s)\right\}}=\sum_{a \in \mathbb{Z}_{p}^{l}} \mathbb{1}_{\left\{g(a, m) \in A_{\epsilon}^{n}(\hat{U} \mid s)\right\}}
$$

Let $Z$ be a uniform random variable over $S^{\prime}$. Then we have

$$
\mathbb{E}\{\theta(s)\}=\sum_{a \in \mathbb{Z}_{p}^{l}} P\left(Z^{n} \in A_{\epsilon}^{n}(\hat{U} \mid s)\right)
$$

we need the following lemmas to proceed:

Lemma III.4. Let $P_{X Y}$ be a joint distribution on $\mathbb{R}^{2}$ and $P_{X}$ and $P_{Y}$ denote its marginals. Let $y$ be a sequence and $Z^{n} a$ random sequence drawn according to $P_{Z}^{n}$. If $D\left(P_{X Y} \| P_{Z} P_{Y}\right)$ is finite then for each $\delta>0$, there exist $\epsilon(\delta)$ and $\bar{\epsilon}(\delta)$ such that if $\epsilon<\epsilon(\delta), \bar{\epsilon}<\bar{\epsilon}(\delta)$ and $y \in A_{\bar{\epsilon}}^{n}\left(P_{Y}\right)$ then

$\limsup \frac{1}{n} \log P_{Z}^{n}\left(\left(Z^{n}, y\right) \in A_{\epsilon}^{n}\left(P_{X Y}\right) \leq-D\left(P_{X Y} \| P_{Z} P_{Y}\right)+\delta\right.$

Proof: This lemma is a generalization of theorem 21 of [14]. The complete proof can be found in a more complete version of this work [19].

Lemma III.5. Let $P_{X Y}$ be a joint distribution on $\mathbb{R}^{2}$ and $P_{X}$ and $P_{Y}$ denote its marginals. Let $y$ be a sequence and $Z^{n}$ a random sequence drawn according to $P_{Z}^{n}$. Then for each $\epsilon, \delta>0$, there exist $\bar{\epsilon}(\epsilon, \delta)$ such that if $y \in A_{\bar{\epsilon}}^{n}\left(P_{Y}\right)$ then

$\liminf \frac{1}{n} \log P_{Z}^{n}\left(\left(Z^{n}, y\right) \in A_{\epsilon}^{n}\left(P_{X Y}\right) \geq-D\left(P_{X Y} \| P_{Z} P_{Y}\right)-\delta\right.$

Proof: This lemma is a generalization of theorem 22 of [14]. The complete proof can be found in [19].

Using these lemmas we get

$$
\mathbb{E}\{\theta(s)\}=p^{k} 2^{-n\left[D\left(P_{\hat{U} S} \| P_{Z} P_{S}\right)+O(\epsilon)\right]}
$$

Similarly, let $Z^{n}=g(a, m)$ and $\tilde{Z}^{n}=g(\tilde{a}, m)$. Note that $Z^{n}$ and $\tilde{Z}^{n}$ are equal if $a=\tilde{a}$ and are independent if $a \neq \tilde{a}$. We 
have

$$
\begin{aligned}
\mathbb{E}\left\{\theta(s)^{2}\right\} & =\sum_{a, \tilde{a} \in \mathbb{Z}_{p}^{l}} P\left(Z^{n}, \tilde{Z}^{n} \in A_{\epsilon}^{n}(\hat{U} \mid s)\right) \\
& =\sum_{a \in \mathbb{Z}_{p}^{l}} P\left(Z^{n} \in A_{\epsilon}^{n}(\hat{U} \mid s)\right) \\
& +\sum_{\substack{a, \tilde{a} \in \mathbb{Z}_{p}^{l} \\
a \neq \tilde{a}}} P\left(Z^{n} \in A_{\epsilon}^{n}(\hat{U} \mid s)\right)^{2} \\
& =p^{k} 2^{-n\left[D\left(P_{\hat{U} S} \| P_{Z} P_{S}\right)+O(\epsilon)\right]} \\
& +p^{k}\left(p^{k}-1\right) 2^{-2 n\left[D\left(P_{\hat{U} S} \| P_{Z} P_{S}\right)+O(\epsilon)\right]}
\end{aligned}
$$

Therefore, using Chebyshev's inequality we obtain

$$
P(\theta(s)=0) \leq \frac{\operatorname{var}\{\theta(s)\}}{\mathbb{E}\{\theta(s)\}^{2}} \leq p^{k} 2^{-n\left[D\left(P_{\hat{U} S} \| P_{Z} P_{S}\right]+O(\epsilon)\right]}
$$

Therefore if $\frac{l}{n} \log p>D\left(P_{\hat{U} S} \| P_{Z} P_{S}\right)$ then the probability of encoding error goes to zero as the block length increases.

2) Decoding Error: The decoder declares error if there is no bin $\mathfrak{B}_{m}$ containing a sequence jointly typical with the channel output $y$ or if there are multiple bins containing sequences jointly typical with $y$. Assume that the message $m$ has been encoded to $x$ according to $W_{X \mid S U}$ where $u=g(a, m)$ and the channel state is $s$. The channel output $y$ is jointly typical with $u$ with high probability. It can be shown [19] that the probability of decoding error is upper bounded by

$$
P_{\text {err }} \leq p^{l} p^{k} 2^{-n\left[D\left(P_{\hat{U} Y} \| P_{Z} P_{Y}\right)+O(\epsilon)\right]}
$$

Hence the probability of decoding error goes to zero if $\frac{k+l}{n} \log p<D\left(P_{\hat{U} Y} \| P_{Z} P_{Y}\right)$. If we choose $\frac{l}{n} \log p$ sufficiently close to $D\left(P_{\hat{U} S} \| P_{Z} P_{S}\right)$ and $\frac{k+l}{n} \log p$ sufficiently close to $D\left(P_{\hat{U} S} \| P_{Z} P_{S}\right)$ we can achieve the rate

$$
\begin{aligned}
R & =\frac{k}{n} \log p \approx D\left(P_{\hat{U} Y} \| P_{Z} P_{Y}\right)-D\left(P_{\hat{U} S} \| P_{Z} P_{S}\right) \\
& =I(\hat{U} ; Y)-I(\hat{U} ; S)
\end{aligned}
$$

\section{B. Arbitrary $U$ and Bounded Continuous Cost Function}

We have shown in Section III-A that for discrete random variables the region given in Theorem III. 1 is achievable. In this part, we make a quantization argument to generalize this result to arbitrary auxiliary random variables. Let $S, U, X, Y$ be distributed according to $P_{S} P_{U \mid S} P_{X \mid U S} W_{Y \mid X}$ where in this case $U$ is an arbitrary random variable. We start with the following theorem:

Theorem III.6. Let $\mathcal{F}_{1} \subseteq \mathcal{F}_{2} \subseteq \cdots$ be an increasing sequence of $\sigma$-algebras on a measurable set $A$. Let $\mathcal{F}_{\infty}$ denote the $\sigma$-algebra generated by the union $\cup_{n=1}^{\infty} \mathcal{F}_{n}$. Let $P$ and $Q$ be probability measures on A. Then

$$
D\left(\left.P\right|_{\mathcal{F}_{n}} \|\left. Q\right|_{\mathcal{F}_{n}}\right) \rightarrow D\left(\left.P\right|_{\mathcal{F}_{\infty}} \|\left. Q\right|_{\mathcal{F}_{\infty}}\right) \text { as } n \rightarrow \infty
$$

where $\left.P\right|_{\mathcal{F}}$ denotes the restriction of $P$ on $\mathcal{F}$.

Proof: Provided in [9] and [1].

For a prime $p>2$ and a real positive number $\gamma$ and for $i=0 \cdots, p-1$ define $a_{i}=\frac{-\gamma(p-1)}{2}+\gamma i$ and define the quantization $Q_{\gamma, p}$ as $Q_{\gamma, p}=\left\{A_{0}, A_{2}, \cdots, A_{p-1}\right\}$ where $A_{0}=\left(-\infty, a_{0}\right], A_{p-1}=\left(a_{p-2},+\infty\right)$ and $A_{i}=\left(a_{i-1}, a_{i}\right]$ for $i=1, \cdots, p-2$. Let the random variable $\hat{U}_{\gamma, p}$ take values from $\left\{a_{0}, \cdots, a_{p-1}\right\}$ according to joint measure

$$
P_{S \hat{U} X Y}\left(\hat{U}=a_{i}, S X Y \in B\right)=P_{S U X Y}\left(U \in A_{i}, S X Y \in B\right)
$$

For all Borel sets $B \subseteq \mathbb{R}^{3}$. For a fixed $\gamma$, let $p \leq q$ be two primes. Then the $\sigma$-algebra induced by $Q_{\gamma, p}$ is included in the $\sigma$-algebra induced by $Q_{\gamma, q}$. Therefore, for a fixed $\gamma$, we can use the above theorem to get

$$
I\left(\left.U\right|_{\mathcal{F}_{\gamma, p}} ;\left.Y\right|_{\mathcal{F}_{\gamma, p}}\right) \rightarrow I\left(\left.U\right|_{\mathcal{F}_{\gamma, \infty}} ;\left.Y\right|_{\mathcal{F}_{\gamma, \infty}}\right) \text { as } p \rightarrow \infty
$$

where $\left.U\right|_{\mathcal{F}_{\gamma, \infty}}$ is a random variable over $Q_{\gamma, \infty}=\left\{A_{i} \mid i \in \mathbb{Z}\right\}$ where $A_{i}=\frac{\gamma}{2}+(\gamma i, \gamma(i+1)]$ with measure $P_{\left.U\right|_{\mathcal{F}_{\gamma, \infty}}}\left(A_{i}\right)=$ $P_{U}\left(A_{i}\right)$.

Let $\gamma_{0}=1$ and define $\gamma_{n}=\frac{1}{2^{n}}$. Note that if $m>n$ then $\mathcal{F}_{\gamma_{n}, \infty}$ is included in $\mathcal{F}_{\gamma_{m}, \infty}$. Also, since dyadic intervals generate the Borel Sigma field ( [15] for example), the restriction of $U$ to the sigma algebra generated by $\cup_{n=1}^{\infty} \mathcal{F}_{\gamma_{n}, \infty}$ is $U$ itself. We can use Theorem III.6 to get

$$
I\left(\left.U\right|_{\mathcal{F}_{\gamma_{n}, \infty}} ;\left.Y\right|_{\mathcal{F}_{\gamma_{n}, \infty}}\right) \rightarrow I(U ; Y) \text { as } n \rightarrow \infty
$$

Combining (8) and (9) we conclude that for all $\epsilon>0$, there exist $\Gamma$ and $P$ such that if $\gamma \leq \Gamma$ and $p \geq \Gamma$ then

$$
\left|I\left(\left.U\right|_{\mathcal{F}_{\gamma, p}} ;\left.Y\right|_{\mathcal{F}_{\gamma, p}}\right)-I(U ; Y)\right|<\epsilon
$$

Since quantization reduces the mutual information $\left(X_{Q} \rightarrow\right.$ $X \rightarrow Y$ ), we have

$$
I\left(\left.U\right|_{\mathcal{F}_{\gamma, p}} ;\left.Y\right|_{\mathcal{F}_{\gamma, p}}\right) \leq I\left(\left.U\right|_{\mathcal{F}_{\gamma, p}} ; Y\right) \leq I(U ; Y)
$$

Therefore $\left|I\left(\left.U\right|_{\mathcal{F}_{\gamma, p}} ; Y\right)-I(U ; Y)\right|<\epsilon$. Also note that $I\left(\left.U\right|_{\mathcal{F}_{\gamma, p}} ; Y\right)=I\left(U_{\gamma, p} ; Y\right)$ since we define the joint measure to be the same. Therefore

$$
\left|I\left(\hat{U}_{\gamma, p} ; Y\right)-I(U ; Y)\right| \leq \epsilon
$$

With a similar argument, $\forall \epsilon>0$ there exist $\gamma$ and $p$ such that

$$
\left|I\left(\hat{U}_{\gamma, p} ; S\right)-I(U ; S)\right| \leq \epsilon
$$

if we take the maximum of the two $p$ 's and the minimum of the two $\gamma$ 's, we can say for all $\epsilon>0$ there exist $\gamma$ and $p$ such that both (10) and (11) happen.

Lemma III.7. The sequence $P_{S \hat{U}_{\gamma_{p}, p} X}$ converges to $P_{S U X}$ in the weak* sense as $p \rightarrow \infty$.

Proof: Provided in a more complete version [19].

The above lemma implies $\mathbb{E}_{P_{S \hat{U}_{\gamma_{p}, p} X}}\{w(X)\}$ converges to $\mathbb{E}_{P_{S U X}}\{w(X)\} \leq W$ since $w$ is assumed to be bounded continuous.

We have shown that for arbitrary $P_{U \mid S}$ and $W_{X \mid S U}$, one can find $P_{\hat{U} \mid S}$ and $W_{X \mid S \hat{U}}$ induced from (7) such that $\hat{U}$ is a discrete variable and

$$
\begin{aligned}
& I(\hat{U} ; Y)-I(\hat{U} ; S) \approx I(U ; Y)-I(U ; S) \\
& \mathbb{E}_{P_{S \hat{U} X}}\{w(X)\} \approx \mathbb{E}_{P_{S U X}}\{w(X)\}
\end{aligned}
$$


Hence, using the result of section III-A, we have shown the achievability of the rate region given in Theorem III. 1 for arbitrary auxiliary random variables when the cost function is bounded and continuous.

\section{Arbitrary $U$ and Continuous Cost Function}

For a positive number $l$, define the bounded random variable $\hat{X}$ by $\hat{X}=\operatorname{sign}(X) \min (l,|X|)$ and let $\hat{Y}$ be distributed according to $W_{\hat{Y} \mid \hat{X}}(\cdot, \hat{x})=W_{Y \mid X}(\cdot, \hat{x})$.

Lemma III.8. As $l \rightarrow \infty, I(U ; \hat{Y}) \rightarrow I(U ; Y)$.

Proof: Proved in a more complete version [19].

Since $\hat{X}$ is bounded and $w$ is assumed to be continuous, $w$ is also bounded. This completes the proof.

\section{Source Coding}

We show the achievability of the rate $R=I(U ; X)-$ $I(U ; S)$ for the Wyner-Ziv problem using nested lattice codes.

Theorem IV.1. For the source $\left(\mathcal{X}, \mathcal{S}, \hat{\mathcal{X}}, P_{X S}, d(\cdot)\right)$ assume $\mathcal{X}, \mathcal{S}, \hat{\mathcal{X}} \subseteq \mathbb{R}$ and $d(\cdot)$ is continuous. Let $U$ be a random variable taking values from the set $\mathcal{U}$ jointly distributed with $X$ and $S$ according to $P_{X S} W_{U \mid X}$ where $W_{U \mid X}(\cdot \mid \cdot)$ is a transition kernel. Further assume that there exists a measurable function $f: \mathcal{S} \times \mathcal{U} \rightarrow \hat{\mathcal{X}}$ such that $\mathbb{E}\{d(X, f(S, U))\} \leq D$. Then the rate $R^{*}(D)=I(X ; U)-I(S ; U)$ is achievable using nested lattice codes.

Here we present a sketch of the proof of this theorem. The complete proof is similar to the channel coding problem and is provided in [19]. The ensemble of codes used for source coding is based on the parity check matrix representation of linear and lattice codes. For a prime number $p$, the linear code over $\mathbb{Z}_{p}$ corresponding to the parity check matrix $H \in \mathbb{Z}_{p}^{k \times n}$ is the kernel of the matrix $H$; i.e. $\mathbb{C}=\left\{u \in \mathbb{Z}_{p}^{n} \mid H u=0\right\}$ where the operations are done mod- $p$. The coset code corresponding to the parity check matrix $H \in \mathbb{Z}_{p}^{k \times n}$ and the bias vector $c \in \mathbb{Z}_{p}^{k}$ is defined as $\mathbb{C}=\left\{u \in \mathbb{Z}_{p}^{n} \mid H u=c\right\}$.

Nested linear codes based on the parity check representation of linear codes can be defined as follows

$$
\begin{aligned}
& \mathbb{C}_{o}=\left\{u \in \mathbb{Z}_{p}^{n} \mid H u=c\right\}, \\
& \mathbb{C}_{i}=\left\{u \in \mathbb{Z}_{p}^{n} \mid H u=c, \Delta H u=\Delta c\right\}
\end{aligned}
$$

where $H \in \mathbb{Z}_{p}^{k \times n}$ and $\Delta H \in \mathbb{Z}_{p}^{k \times n}$. The ensemble of lattice codes are then constructed using (1) and (4). The encoding and decoding rules are as follows: For $m \in \mathbb{Z}_{p}^{k}$, Let $\mathfrak{B}_{m}$ be the $m$ th bin of $\Lambda_{i}$ in $\Lambda_{o}$. The encoder observes the source sequence $x \in \mathcal{X}^{n}$ and looks for a vector $u$ in the outer code $\Lambda_{o}$ which is typical with $x$ and encodes the sequence $x$ to the bin of $\Lambda_{i}$ in $\Lambda_{o}$ containing $u$. The encoder declares error if it does not find such a vector.

Having observed the index of the bin $m$ and the side information $s$, the decoder looks for a unique sequence $u$ in the $m$ th bin which is jointly typical with $s$ and outputs $f(u, s)$. Otherwise it declares error. Similar to the channel coding problem, this theorem is first proved for discrete auxiliary random variables and then it is generalized to arbitrary sources.

\section{Conclusion}

We have shown that nested lattice codes are optimal for the Gelfand-Pinsker problem as well as the Wyner-Ziv problem.

\section{REFERENCES}

[1] A. R. Barron. Limits of Information, Markov Chains, and Projection. Proceedings of IEEE International Symposium on Information Theory, 2000. Sorrento, Italy.

[2] R. De Buda. Some optimal codes have structure. IEEE Journal on Selected Areas in Communications, 7:893-899, 1989.

[3] I. Csiszar. Linear Codes for Sources and Source Networks: Error Exponents, Universal Coding.

[4] U. Erez and R. Zamir. Achieving $\frac{1}{2} \log (1+$ SNR) on the AWGN Channel With Lattice Encoding and Decoding. IEEE Transactions on Information Theory, 50:2293-2314, 2004.

[5] U. Erez and R. Zamir. Capacity and Lattice Strategies for Canceling Known Interference. IEEE Transactions on Information Theory, 51:3820-3833, 2005.

[6] U. Erez and R. Zamir. Lattices Which Are Good for (Almost) Everything. IEEE Transactions on Information Theory, 51:3401-3416, 2005.

[7] T. Gariby and U. Erez. On General Lattice Quantization Noise Proceedings of IEEE International Symposium on Information Theory, 2008. Toronto, Canada.

[8] S. I. Gelfand and M. S. Pinsker. Coding for channel with random parameters. Problems of Control and Information Theory, 9:19-31, 1980.

[9] P Harremos and K Khler Holst. Convergence of Markov Chains in Information Divergence. Journal of Theoretical Probability, 22(1):186202, 2011.

[10] J. Korner and K. Marton. How to encode the modulo-two sum of binary sources. IEEE Transactions on Information Theory, IT-25:219-221, Mar. 1979.

[11] D. Krithivasan and S. S. Pradhan. Distributed source coding using abelian group codes. 2011. IEEE Transactions on Information Theory(57)1495-1519.

[12] T. Linder and C. Schlegel. Corrected Proof of de Buda's Theorem. IEEE Transactions on Information Theory, 39:1735-1737, 1993.

[13] H. A. Loeliger. Averaging bounds for lattices and linear codes. IEEE Transactions on Information Theory, 43:1767-1773, 1997.

[14] P. Mitran. Typical Sequences for Polish Alphabets. 2010. Online: http://arxiv.org/abs/1005.2321.

[15] P. Mrters, O. Schramm Y. Peres, and W. Werner. Brownian Motion. Cambridge University Press, 2010.

[16] B. A. Nazer and M. Gastpar. Computation over multiple-access channels. IEEE Trans. on Inf. Th., 53, Oct. 2007.

[17] A. Padakandla and S. S. Pradhan. Nested linear codes achieve martons inner bound for general broadcast channels. Proc. IEEE Int. Symp. Information Theory, 2011. Saint Petersburg, Russia.

[18] T. Philosof, A. Kishty, U. Erez, and R. Zamir. Lattice strategies for the dirty multiple access channel. Proceedings of IEEE International Symposium on Information Theory, July 2007. Nice, France.

[19] A. G. Sahebi and S. Sandeep Pradhan. Nested lattice codes for arbitrary continuous sources and channels. 2012. Online: http://arxiv.org/submit/410552.

[20] S. Sridharan, A. Jafarian, S. Vishwanath, S. A. Jafar, and S. Shamai. A layered lattice coding scheme for a class of three user gaussian interference channels. 2008. Online: http://arxiv.org/abs/0809.4316.

[21] R. Urbanke and B. Rimoldi. Lattice Codes Can Achieve Capacity on the AWGN Channel. IEEE Trans. on Inf. Th., 44:273-278, 1998.

[22] A. Wyner. The rate distortion function for source coding with side information at the decoder-ii. Information and Control, 38:60-80, 1978.

[23] A. Wyner and J. Ziv. The rate distortion function for source coding with side information at the decoder. IEEE Transactions on Information Theory, 22:1-10, 1976.

[24] R. Zamir and M. Feder. On lattice quantization noise. IEEE Transactions on Information Theory, 42:1152-1159, 1996.

[25] R. Zamir and S. Shamai. Nested linear/lattice codes for wyner-ziv encoding. ITW, 1998. Ireland.

[26] R. Zamir, S. Shamai, and U. Erez. Nested linear/lattice codes for structured multiterminal binning. IEEE Transactions on Information Theory, 48(6):1250-1276, 2002. 